\title{
CONSIDERATIONS ON LIQUID-CHROMATOGRAPHIC SEPARATION FOR AN EQUIMOLAR MIXTURE OF 2,4-DINITROPHENYLHYDRAZONES OF ACETALDEHYDE AND DIACETYL
}

\author{
Gheorghe Zgherea \\ Department of Chemistry, Faculty of Sciences, University "Dunărea de Jos" of Galați \\ 111 Domnească street, 800.201 Galați, Romania, phone +40236 414871, fax +40236461353 \\ gzgherea@chem.ugal.ro
}

\begin{abstract}
An equimolar mixture of 2.4-dinitrophenylhidrazones (2.4DNPH-ones) providing by acetaldehyde and diacetyl must be analyzed by liquid-chromatographic separation, using the mechanism of repartition with reverse phase; that full papers is for identification the optimal analytical conditions. As mobile phase are utilized various binary mixtures eluent, containing water and methanol, with $0-45 \%$ water. By the experimental studies were identified four domains of behavior and two optimal binary mixtures, with $25 \%$ and $45 \%$ water, thus this is a study on the behavior of binary mixtures mobile phases. The peaks are characterized by values of retention times and by position. The separation processes were appreciated by difference between the retention times of peaks; if the percent of water increase, the values of retention times is higher. When the percent of water is $45 \%$, the difference between the retention times is maxim, associated with a change of peaks position.
\end{abstract}

Keywords: acetaldehyde, diacetyl, reverses phase, polarity, percent of water, inversion of position

\section{Introduction}

Any foods obtained by fermentation have small quantities of carbonyl compounds. They have a very important contribution to the flavor and the fragrance; between these compounds there are acetaldehyde and diacetyl. On consider that the diacetyl is the vicinal dicetone with a very important contribution at the sensorial properties of beer. In addition, his concentration in beer $\left(0.01-0.2 \mathrm{mg} \cdot \mathrm{L}^{-1}\right)$ is a reference values for the level of oxidative process during preparative process. That explains the major preoccupations on the physical-chemistry methods to identification and to dose beer's diacetyl. Between these methods there is the distillation of carbonyl compounds and transfer in a strong acid solution of 2.4-dinitrophenylhidrazine; the mixture of insoluble precipitates of 2.4-DNPH-ones is separated by filtration, washed with bidistilate water, dried and solved in organic solvent (methanol, acetonitrile, tetrahydrofuran), followed by an appropriate liquid-chromatographic separation.

The described analytical method may be use for any mixture of carbonyl compounds. The 2.4-DNPH-ones are solid substances, yellows, soluble in organic solvents [1]. The literature doesn't have specific reference about their polarity [2-3]; may be compared only the polarity values for carbonyl compounds providers.

After their behaviors, the mixtures of 2.4-DNPH-ones may be analyzed by HPLC, using the mechanism of repartition with reverse phase; may be used a slightly polar stationary phase (octadecysilan) and a mixture of mobile phase containing water (strongly polar solvent) with a slightly organic solvent [4-7]. The no polar stationary phase assures a strongly retaining of slightly polar molecules of 2.4DNPH-ones. The no polar mobile phases provides a better solubilisation of 2.4DNPH-ones, assuring a better mass transfer between the mobile and stationary phase; thus, the peak are symmetrically, narrows and with small values of retention times. The polar mobile phase provides a small solubilisation and a hard mass transfer between the phases; the peaks are asymmetrically and with higher values of retention times. Therefore, a higher resolution between peaks is assured only by optimal mixture of mobile phase, when the difference between retention time values is maxim, for the adjacent peaks.

In the upper case, analytical difficulties are generating by the similar behavior of $2.4 \mathrm{DNPH}$-ones providing by diacetyl (2.4-DNPD) and acetaldehyde (2.4-DNPHAA). Must be established the optimal mixture of mobile phase (with water and methanol) to assure the best separation for an equimolar mixture (a model synthetic mixture) of the two 2.4-DNPH-ones.

\section{Results}

Ten chromatograms were obtained. Each chromatogram has two chromatographic signals (peak); the identity of signals was verified by addition method.

The behavior of eluent (methanol or mixtures of mobile phase) was appreciate by difference between the values of retention times of 2.4DNPH-ones 


$$
\triangle R T=\left(R T_{2,4 D N F H A A}-R T_{2,4 D N F H D}\right)
$$

The $\Delta \mathrm{RT}$ values are dependence by molecular structure of $2.4 \mathrm{DNPH}$-ones and the phenomena that are produce during the liquid chromatographic separation process, being an expression of relation between their repartition coefficients values.

The $\Delta \mathrm{RT}(\mathrm{sec})$ values are represented graphic against the mobile phase polarity, defined by the percent of water value in the mixture of mobile phases. On obtain the diagram of figure 1, with delimitation of four especially domains behavior of eluent mixtures.

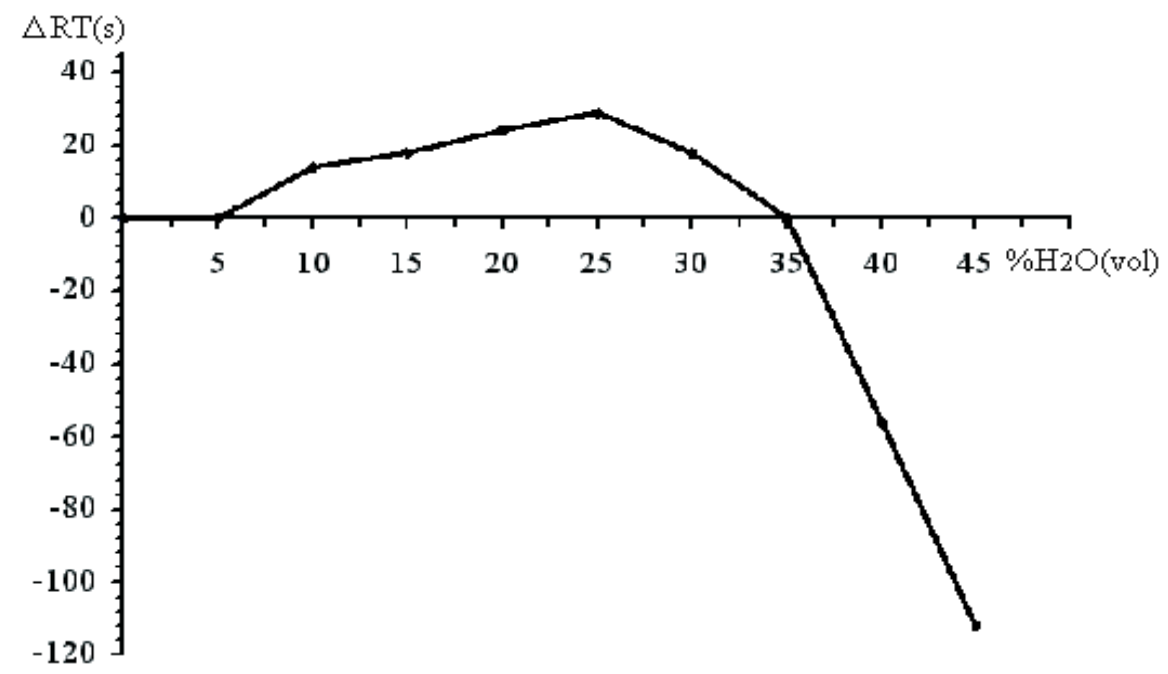

Figure 1 Diagram of $\Delta \mathrm{RT}$ values against the polarity of mobile phase mixture

\section{Discussion}

Need to explain the structure and behavior of two 2.4DNPH-ones, beginning from the structure of provider's carbonyl compounds. To explain the difference between behaviors, need to consider physical behavior of two species of molecules: mass, volume and polarity. Can be considerate that the asymmetric molecules of $2.4 \mathrm{DNPHAA}\left(\mathrm{M}_{2,4 \mathrm{DNFHA}}=\right.$ $\left.224 \mathrm{~g} \cdot \mathrm{mol}^{-1}\right)$ are slowly plus polar than the symmetric molecules of $2.4 \mathrm{DNPHD}\left(\mathrm{M}_{2,4 \mathrm{DNFHD}}=446 \mathrm{~g} \cdot \mathrm{mol}^{-1}\right)$.

To explain the four distinct behaviors of the mobile phase mixtures, need to considerate the contributions of the essential process which there are on the stationary phase: the zone of concentration, for each 2.4DNPH-one, is in extension. This process affects the retention time values, thus and the $\Delta R T$ values.

On the stationary phase there are, simultaneously, three distinct phenomena: the longitudinal diffusion, the turbulent diffusion and the no local equilibrium (resistance at the mass transfer process, between the two immiscible phases). On may be considered that only two distinct phenomena have the most important contribution at the concentrated zone extension. The stationary phase have only spherically and equal particles and the mobile phase flow at the high pressure (> $100 \mathrm{bar}$ ); in this instance, the contribution of turbulent diffusion is insignificant for the general process.

Follow-up are presented the behavior of mobile phase, against the water percent in the mixture.

\section{The domain 0-5\% water (the mobile and stationary phase are no polar too)}

The polarity of mixture is very slow, as the stationary phase as. The interaction between the 2.4DNPH-one's molecules and the stationary phase must be higher, but the most important part of molecules is dissolved in the no polar liquid mobile phase. No local equilibrium is completely absent, but is very weak the phenomenon of longitudinal diffusion, generating a simultaneously elution for the molecules of the two 2.4DNPH-ones. The two molecular species are characterized by identically and low coefficients of repartition and retention times values, thus $\triangle R T=0$ seconds.

\section{The domain $5-25 \%$ water (the behavior's molecules is in accord with their dimensions)}

Increase the polarity of the eluent by water percent; this value produces an increase of interaction between the 2.4DNPH-one's molecules and no polar stationary phase. Simultaneously, increase the intensity of no local equilibrium.

The lower and asymmetrically molecules of 2.4DNPHAA, weak polar, are characterized by lower value of diffusion coefficient from the stationary phase to mobile phase, being intense retained. The lower diffusion process 
in mobile phase produce an intense process of longitudinal diffusion, thus an extension of zone; the 2.4DNPHAA molecules generate large peaks, symmetrically, but with high values of retention time.

The big and symmetrically molecules of 2.4DNPHD, no polar or slightly polar, are characterized by higher value of diffusion coefficient from the stationary to mobile phase. Because their physical dimension, these molecules are slowly values of longitudinal diffusion in the mobile phase, generating narrow and symmetrically peak, but with low values of retention time. Like the different behavior, the relation between the coefficients of repartition is

$$
k_{2.4 D N P H A A}>k_{2.4 D N P H D}
$$

The retention time values there are in the same relation, thus the $\Delta R T$ values are positive; the mobile phase with $25 \%$ water represent the binary eluent mixture who generate two chromatographic peaks with a maxim value of difference between retention time values, $\Delta R T=29$ seconds.

\section{The domain $25-35 \%$ water (the behavior's molecules is in accord with their slow polarity)}

For higher values of water percent, the polar eluent produce a higher interaction of molecules with the particle of stationary phase, increasing the values of retention times. The phenomenon of longitudinal diffusion becomes important. In addition, became very intense the process of no local equilibrium (resistance at mass transfer from the stationary phase to the mobile phase); the eluent mixture with a high percent of water undertake very hard the organic molecules of 2.4DNPH-ones. Conclusively, increase the dimension's zone of concentration and the values of retention times. The values of retention times have an unequal increasing, with the tendencies to become identically (at higher value of water percent), thus the molecular species have the same behavior; the $\Delta R T$ values subside. If the mobile phase have $30 \%$ water, the corresponding value $\Delta R T=18$ seconds. For the superior limit of domain, $35 \%$ water, the two $2.4 \mathrm{DNPH}$-ones are characterized by identical values of repartition coefficients, thus, equal values of retention times, $\Delta R T=0$ seconds

$$
k_{2,4 D N F H A A}=k_{2,4 D N F H D}
$$

All the mixture of mobile phase with 25-35\% water (plus polar mixture) produce subside of peak's resolution.

\section{The domain $35-45 \%$ water}

The eluent mixtures become very polar and produce an intense interaction between the non polar molecules and the particle of stationary phase, non polar too; the behavior of molecules may be justified again by the slowly different values of polarity. In this case, the values of repartition coefficients increase very much; for the phenomenon of mass transfer from stationary phase to mobile phase there are a similarly evolution, extending the zones of concentration. Simultaneously, the retention time's values increase with proper rate. On observe that relation between the values of repartition coefficients become,

$$
k_{2,4 D N F H A A}<k_{2,4 D N F H D}
$$

A similar relation is between retention times values, thus $\triangle R T$ values become negative.

Because the water percent is very higher, the $2.4 \mathrm{DNPH}$-ones molecules transport is affected by no local equilibrium and by high values of coefficient of longitudinal diffusion.

The symmetric and no polar molecules of 2.4DNPHD are strongly retained on stationary phase particles. The asymmetric and weak polar molecules of 2.4DNPHAA pass soft from the particles of stationary phase in the liquid polar mobile phase.

The polar mixture mobile phase provides an inversion of zones position on stationary phase, thus a position's inversion of the peaks in chromatogram. The eluent mixture with $40 \%$ water assure a separation process characterized by a good resolution between peak, $\Delta R T=-56$ seconds; if the percent of water is $45 \%$, the process of chromatographic separation have a better resolution, $\Delta R T=-112$ seconds.

\section{Conclusion}

On the experimental results may be formulated the following conclusions:

1. Using binary isocratic mixture, containing methanol and water $(0-45 \%$, percent of volume), the equimolar mixture of 2.4DNPHAA and 2.4DNPHD may be separate by liquid-chromatography with reverse phase; the resolution of chromatographic signals depend by the polarity of mobile phase.

2. The similarly behavior of 2.4DNPH-ones may be described by structurally particularity of carbonyl compounds provider: dimension, symmetry, solubility in solvents and, especially, by polarity.

3. The succession of concentrated zones on the stationary phase, thus the positions of signals in chromatograms 
is de pendent of mobile phase polarity; the retention time values of two 2.4DNPH-ones are too higher, for big values of mobile phase polarity.

4. Higher values of mobile phase polarity provide a change of chromatographic signal positions, thus the inversion of signals positions in chromatograms.

\section{Experimental}

Etalons, solvents, equimolar mixture, mobile phase and liquid chromatographic separations

The 2.4DNPH-ones etalons, provided by acetaldehyde (2.4DNPHAA) and diacetyl (2.4DNPHD), was synthesized (by simply mixing at room temperature, with a better yield, being perfectly reproducible) and purified (by recristalisation) in our laboratory, through: filtration, washing with water, drying and filtration.

In acetonitrile (Merck) was obtained solution $10^{-4} \mathrm{M}$, for each 2.4DNPH-one etalon. In figure 2 are presented the stuctures of two 2.4DNPH-ones etalons. The equimolar mixture was obtained by mixing the two etalon solutions in ratio $1: 1(\mathrm{v}: \mathrm{v})$; each $2.4 \mathrm{DNPH}$-one has $5 \cdot 10^{-5} \mathrm{M}$ concentration.

The bidistilate water $\left(2 \mu \mathrm{S} \cdot \mathrm{cm}^{-1}\right)$ is prepared in our laboratory, from distillated water, after a treatment with sulphuric acid and kalium permanganate. The mobile phases are methanol and nine binary mixtures containing bidistilate water and methanol (Merck, for liquid chromatography); the percent of water has the value $0-45 \%$, controlled by LC gradient programmer module.

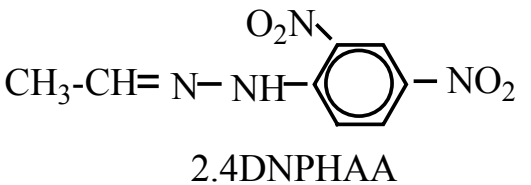

2.4DNPHAA

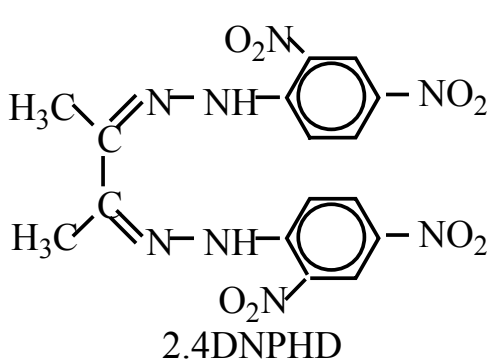

Figure 2 The chemical structures of 2.4DNPH-ones etalons

\section{Apparatus}

The separations was utilized LC-XPD Pye Unicam Philips liquid chromatograph who include: solvent metering system (two reservoir for mobile phases, the module of controlled mixing type LC gradient programmer, high pressure pump type Spectra Physics 100A), Rheodyne valve 7125 (for the mixture sample), liquid chromatographic column (Spherisorb 5ODS, $25 \mathrm{~cm}$ length and 4,6 mm internal diameter, with gradient of stationary phase), with a module of thermostatation, LC-UV detector, electronic integrator for chromatographic signal (type DP101) and potentiometric recorder (type PM8251).

\section{Experimental conditions}

The equimolar mixture was liquid-chromatographic separate, in the following conditions:

$10 \mu \mathrm{L}$, volume of mixture;

Temperature of separation column $37,5^{\circ} \mathrm{C}$ (at high temperature of mobile phase increase the solubility of compounds);

Detector: UV Absorbance $(\lambda=355 \mathrm{~nm})$;

Flow rate $=1 \mathrm{~mL} \cdot \mathrm{min}^{-1}$.

Liquid chromatographic separations

Identical volumes of equimolar mixture were separate by HPLC, using the précised mobile phases.

\section{References}

[1] Gheorghe Zgherea, Synthesis and Comparative Characterization of Eight 2,4-dinitrophenylhydrazones, The Annals of „Dunărea de Jos” University of Galați, Fascicle VI, 1999, ISSN 1221-4574, pp 4-9.

[2] David R. Lide, ed., CRC Handbook of Chemistry and Physics, Internet Version 2005, <http://www.hbcpnetbase. com>, CRC Press, Boca Raton, FL, 2005.

[3] Zahn C. T., The Dielectric Constant of Dichlorethane, Dibromethane, Chlorobromethane, and Diacetyl; and the Phenomenon of Free Rotation, 1932 The American Physical Society, http://link.aps.org/abstract/PR/v40/p2

[4] Candin Liteanu; Simion Gocan; T. Hodisan; H. Nascu, Cromatografia de Lichide, Editura Stiintifică, Bucureşti, 1974, pp 156-157.

[5] Douglas A. Skoog, Principles of Instrumental Analysis, Third edition, Saunders College Publishing, 1985, pp 801-815.

[6] Simion Gocan, Cromatografie de Înaltă Performanță, Partea a II-a, Cromatografia de Lichide pe Coloane, Editura RISOPRINT, Cluj-Napoca, 2002, pp 173-183.

[7] Raymond P.W. Scott, Liquid Chromatography, pag 55-62, http: //www.library4science.com/eula.html. 